\title{
On the interactions of turbulent convection and rotation in $\mathrm{RGB}$ stars
}

\author{
Ana Palacios ${ }^{1}$ and Allan S. Brun ${ }^{1}$ \\ ${ }^{1}$ CEA/DSM/DAPNIA/Service d'Astrophysique and AIM UMR 7158, CE Saclay Bât 709, \\ F-91191 Gif-sur-Yvette, France \\ email: ana.palacios@cea.fr or sacha.brun@cea.fr
}

\begin{abstract}
We have performed the first three-dimensional non-linear simulation of the turbulent convective envelope of a rotating $0.8 \mathrm{M}_{\odot}$ RGB star using the ASH code. Adopting a global typical rotation rate of a tenth of the solar rate, we have analyzed the dynamical properties of the convection and the transport of angular momentum within the inner $50 \%$ in radius of the convective envelope. The convective patterns consist of a small number of large cell, associated with fast flows $(\sim 3000 \mathrm{~m} / \mathrm{s})$ and large temperature fluctuations $(\sim 300 \mathrm{~K})$ in order to carry outward the large luminosity $\left(L \sim 400 \mathrm{~L}_{\odot}\right)$ of the star. The interactions between convection and rotation give rise to a large radial differential rotation and a meridional circulation possessing one cell per hemisphere, the flow being poleward in both hemisphere. By analysing the redistribution of angular momentum, we find that the meridional circulation transports the angular momentum outward in the radial direction, and poleward in the latitudinal direction, and that the transport by Reynolds stresses acts in the opposite direction. From this 3-D simulation, we have derived an average radial rotation profile, that we will ultimately introduce back into 1-D stellar evolution code.
\end{abstract}

Keywords. Convection, rotation, hydrodynamics, stars: evolution, stars: interiors

\section{Astrophysical context}

The standard $\dagger$ stellar evolution theory predicts that the surface chemical composition of low-mass stars is modified after the end of the main sequence (turn-off) by the deepening of the convective envelope (first dredge-up), and is kept unaltered after the first dredge-up completion until they reach the horizontal branch.

On the other hand, observations paint a rather different picture, indicating that not less than $98 \%$ of low-mass red giant branch (hereafter RGB) stars exhibit surface abundance variations of lithium, carbon and nitrogen on the upper part of the RGB (Charbonnel \& do Nascimento 1998). These "abundance anomalies" appear at the bump, which is the evolutionary point where the outgoing hydrogen burning shell crosses and erases the chemical (mean molecular weight) discontinuity left behind by the retreating convective envelope after the first dredge-up. They are the result of hydrogen nuclear burning through the CNO cycle, and have been demonstrated to be clear signatures of internal transport processes (Gratton et al. 2000). The nature of such internal transport processes has been related to rotation since the pioneering work by Sweigart \& Mengel (1979) (see also Charbonnel 1995), but it is only up to recently that consistent treatment of rotationinduced mixing by meridional circulation and shear turbulence was applied to low-mass stellar evolution on the RGB. Using the best available tools to describe rotational mixing, in Palacios et al. (2006) we found that meridional circulation and shear-induced turbulence, as described by the present formalism, and taken as "stand alone", do not drive

$\dagger$ By this we refer to models of non-rotating, non-magnetic stars, in which convection and atomic diffusion are the only transport processes considered. 
sufficient mixing in the radiative zone so as to modify the surface chemical composition of RGB stars. One of the weaknesses of the formalism we used is to consider solid-body rotation in the convective zones. The analysis of the rotation of horizontal branch stars in globular clusters recently led Sills \& Pinsonneault (2000) to suggest, as Sweigart \& Mengel (1979) did, that in order to reproduce the rotation of HB stars, a large amount of angular momentum should be retained in the inner part of the stars during the RGB ascension. This can be achieved if RGB convective envelopes rotate differentially. After confirming in Palacios et al. (2006) that assuming conservation of the specific angular momentum $\left(\Omega(r) \propto r^{-2}\right)$ favours turbulent mixing below the convective zone, we have decided to investigate more deeply the interaction of turbulent convection and rotation in such an extended convective zone. To do so, we turned to 3D simulations, that can be used as numerical experiments in order to better understand such dynamical interactions.

\section{Numerical simulation : a brief description}

We have used the anelastic spherical harmonic (ASH) code in its hydrodynamic mode to study in details the interaction of turbulent convection and rotation in the inner part of the convective envelope of a low-mass RGB star. The reader is referred to Brun \& Toomre (2002) and Brun et al. (2004) for details on the code. Let us here briefly summarize the main characteristics of our simulation. We constructed the $3 \mathrm{D}$ simulation by using as initial reference state, a 1D model of a RGB star at the bump with the following characteristics : $\mathrm{M}_{\mathrm{ini}}=0.8 M_{\odot}, \mathrm{Z}=210^{-4}, \mathrm{~L}_{*}=425 L_{\odot}$ and $\mathrm{R}_{*}=40 R_{\odot}$. In order to reduce the density contrast to an amplitude that can be numerically handled by ASH, we reduced the computational domain to the inner $50 \%$ of the convective envelope, in the region $\mathrm{r} \in\left[0.05 R_{\odot} ; 0.5 R_{\odot}\right]$, where the luminosity is constant and $\Delta \rho=100\left(\rho \in\left[10^{-5} ; 10^{-3}\right] \mathrm{g} . \mathrm{cm}^{-3}\right)$. We assume as initial solid-body rotation $\Omega=\Omega_{\odot} / 10=2.610^{-7} s^{-1}$. We assume rigid stress free boundary conditions at the edges of the computational domain, and present here first results for a simulation with a Prandtl number $P_{r}=\nu / \kappa=1$, and a Reynolds number $R_{e}=v L / \nu \approx 500$. We impose a flux of radiative energy at the base of our domain that is extracted at the surface. The simulation has been evolved over 3230 days, which represent more than 10 rotations of the system.

\section{Convective patterns in the inner part of the extended convective envelope of a red giant star}

The convective instability sets in rapidly, with a linear growth phase spanning 130 days after the beginning of the simulation, and non-linearly saturates, reaching a statistical equilibrium that lasts for the remaining of the simulated time. The resulting convective pattern is represented in Fig. 1 : it consists of large blobs covering the top of the simulation domain, the size of which decreases towards the bottom of the domain, partly due to a geometrical effect. The downflows are associated with narrower and cooler structures. There is a clear correlation between radial velocity and temperature at all depths. The characteristic time for convective overturning is about 150 days, which is of the order of half the rotation period. The large luminosity, $400 \mathrm{~L}_{\odot}$, associated with the radiative flux that is applied at the base of the domain is transported through the spherical shell thanks to the convective (enthalpy) flux associated with the very large fluctuations of the radial velocity $(\approx 3000 \mathrm{~m} / \mathrm{s})$ and of the temperature $(\approx 300 \mathrm{~K})$.

Contrary to simulations under Boussinesq approximation, we obtain asymmetric convection, with large slow upflows and narrow fast downflows, characteristic of compressible 


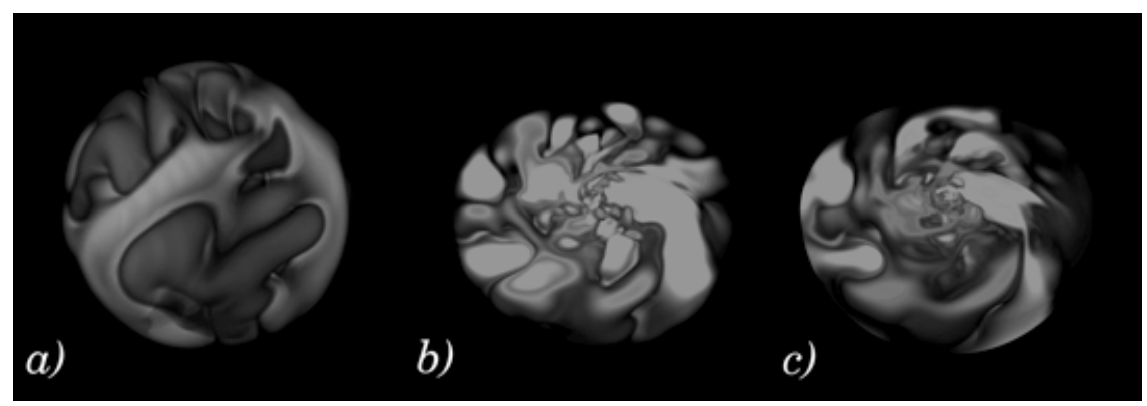

Figure 1. Global volume rendering of the radial velocity flow in a full sphere view at the top of the computational domain $(a)$, and rendering of the radial velocity $(b)$ and the temperature (c) with the northern hemisphere being removed, such as to display the equatorial and inner regions. Dark grey patterns are warm upward flows and light grey patterns are cool downward flows.

computations. The enthalpy flux is very large and represents more than $170 \%$ of the imposed radiative flux (i.e convective luminosity $\mathrm{L}_{e n}=4 \pi r^{2}\left(\bar{\rho} \mathrm{Cp}<v_{r}{ }^{\prime} \mathrm{T}>\right.$ ) $\left.=1.7 \mathrm{~L}_{*}\right)$ in the inner half part of the computational domain. In this region, it compensates the large negative kinetic energy flux, which can be there as high as $70 \%$ the imposed radiative flux. This is in sharp contrast with MLT modelling used in 1-D models, that assumes that the convective luminosity is equal to the stellar luminosity, and that the kinetic energy luminosity is zero.

\section{Established differential rotation and angular momentum balance}

In this extended convective domain, strong differential rotation develops, as can be seen from Fig. 2.a, where we display the temporal (over 1800 days) and longitudinal average of the angular velocity profile realized in the simulation. Due to the moderate degree of turbulence in the simulation, the obtained rotation is very cylindrical. A large differential rotation is achieved both in the radial and latitudinal directions. The amplitude of the differential rotation in the radial direction is $\left(\Delta \Omega^{r} / \Omega_{0}\right)_{e q}=\left(\Omega_{R_{t o p}}-\Omega_{R_{b o t}} / \Omega_{0}\right)=7.5$, the inner prograde regions rotating faster than the outer retrograde ones. The contrast in angular velocity in latitude is of $\left(\Delta \Omega^{\theta} / \Omega_{0}\right)_{t o p}=\left(\Omega_{\theta=0}-\Omega_{\theta=\pi / 2} / \Omega_{0}\right)=1.2$ (where $\theta$ is the colatitude), the high latitude regions rotating faster than the equator. The rotation that sets in is also anti-solar, with a slow equator and fast poles.

The associated meridional flows are represented in Fig. 2.b. They exhibit one large poleward cell at the surface per hemisphere, with a return flow deeper down.

Meridional circulation contributes to a non-negligible part of the total kinetic energy (KE), which is of the order of a few $10^{6} \mathrm{erg} / \mathrm{cm}^{3}$. Half of it corresponds to the nonaxisymmetric convection (CKE). The kinetic energy associated with differential rotation (DRKE) represents $40 \%$ of the total energy while the $10 \%$ remaining are associated with meridional circulation (MCKE; see Brun \& Toomre 2002 for their analytical expression). This energy distribution is very different from that obtained for simulations of the convective envelope of the Sun, where MCKE represents less than $1 \%$ of the total kinetic energy, and exhibits a multi-cellular structure, both in radius and latitude. The moderate degree of turbulent achieved in this simulation can perhaps explain the rather simple meridional flow established.

In our simulation, the choice of stress-free boundaries at the edges of the computational domain results in the conservation of the angular momentum. The $\phi$-component of the 


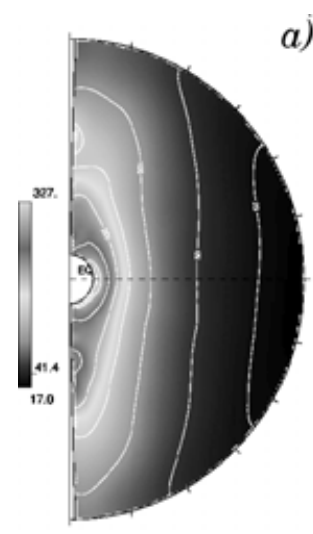

a)

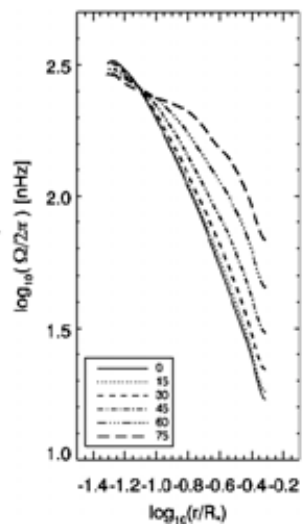

b)

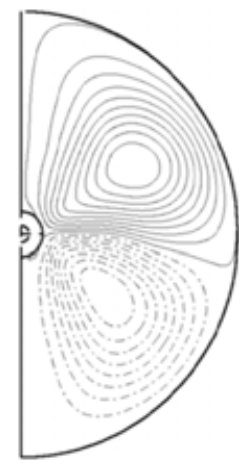

Figure 2. (a) Temporal and longitudinal averages of the angular velocity profile achieved in the simulation formed over 1800 days. Radial cuts at indicated latitudes are presented on a logarithmic scale in the middle panel. (b) Streamlines of the mean axisymmetric meridional circulation achieved in our simulation, averaged over 1800 days. Solid contours denote counterclockwise circulation. The case studied here presents one poleward cell per hemisphere.

momentum equation expressed in conservative form and averaged in time and longitude yields

$$
\frac{1}{r^{2}} \frac{\partial\left(r^{2} \mathcal{F}_{r}\right)}{\partial r}+\frac{1}{r \sin \theta} \frac{\partial\left(\sin \theta \mathcal{F}_{\theta}\right)}{\partial \theta}=0
$$

involving the mean radial angular momentum flux

$$
\mathcal{F}_{r}=\hat{\rho} r \sin \theta[\underbrace{-\nu r \frac{\partial}{\partial r}\left(\frac{\hat{v}_{\phi}}{r}\right)}_{\mathcal{F}_{r, V}}+\underbrace{\widehat{v_{r}^{\prime} v_{\phi}^{\prime}}}_{\mathcal{F}_{r, R}}+\underbrace{\hat{v}_{r}\left(\hat{v}_{\phi}+\Omega_{0} r \sin \theta\right)}_{\mathcal{F}_{r, M}}]
$$

and the mean latitudinal angular momentum flux

$$
\mathcal{F}_{\theta}=\hat{\rho} r \sin \theta[\underbrace{-\nu \frac{\sin \theta}{r} \frac{\partial}{\partial \theta}\left(\frac{\hat{v}_{\phi}}{\sin \theta}\right)}_{\mathcal{F}_{\theta, V}}+\underbrace{\widehat{v_{\theta}^{\prime} v_{\phi}^{\prime}}}_{\mathcal{F}_{\theta, R}^{\prime}}+\underbrace{\hat{v}_{\theta}\left(\hat{v}_{\phi}+\Omega_{0} r \sin \theta\right)}_{\mathcal{F}_{\theta, M}}] .
$$

$\mathcal{F}_{r, V}$ and $\mathcal{F}_{\theta, V}$ are related to the angular momentum flux due to viscous transport, $\mathcal{F}_{r, R}$ and $\mathcal{F}_{\theta, R}$ are related to the transport through Reynolds stresses and $\mathcal{F}_{r, M}$ and $\mathcal{F}_{\theta, M}$ represent the angular momentum flux due to meridional circulation.

In Fig 3.a, we have represented these different contributions to the radial and latitudinal fluxes by integrating over concentric shells and cones with different angles respectively, and by subsequently averaging over the last 1800 days of the simulation. As it can be seen from the solid lines representing the total flux, the simulation is now balanced, since this line is near zero. The radial viscous flux is small and positive, this sign being expected from the angular velocity gradient. The transport by meridional circulation and Reynolds stresses are of opposite signs and balance each other in radius and latitude. The meridional circulation radial flux is positive, which indicates an outward transport of angular momentum, whereas in latitude it is negative in the northern hemisphere, corresponding to a poleward transport of angular momentum. 

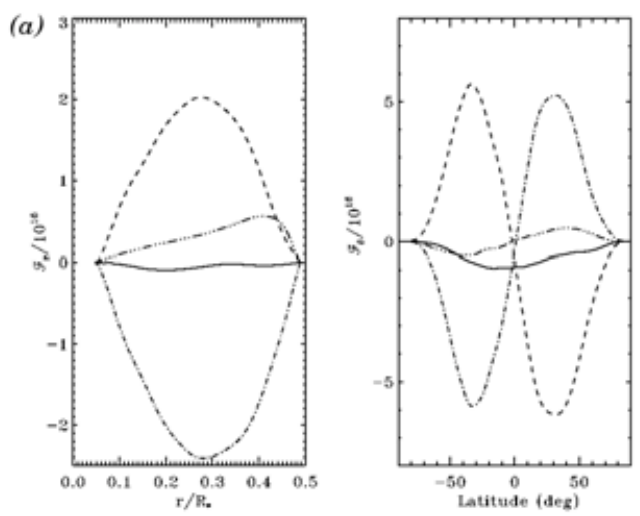

(b)

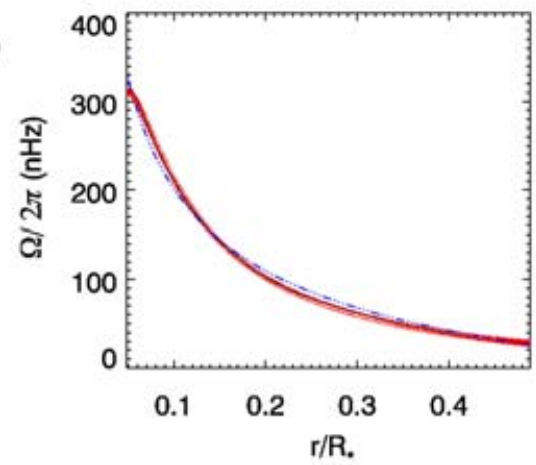

Figure 3. (a) Temporal average of the latitudinal line integral of the angular momentum flux $\mathcal{F}_{r}$ (divided by $R^{2}$ ), and of the radial line integral of the angular momentum flux $\mathcal{F}_{\theta}$. The fluxes are decomposed into their viscous (dashed-double dotted lines), Reynolds stress (dot-dashed lines), and meridional circulation (long dashed lines) components. The solid curves represent the total fluxes and serve to indicate the quality of the stationarity achieved in the simulation. The fluxes have been averaged over a period of 1800 days. (b) Collapsed angular velocity profile along the radial direction for a temporal average of 1800 days. The double-dotted dashed line represents a $10 \%$ quality fit (see text) and the squares represent a better quality ( $2 \%$ at worse) fit achieved using a ratio of polynoms.

In Fig. 3.b, we present the collapsed angular velocity profile obtained by temporal and angular (latitudinal and longitudinal) average of the $v_{\phi}$ component of the velocity over more than 10 rotation periods (e.g. 3230 days).

The resulting profile confirms the strong differential rotation seen at all latitudes in Fig. 2.a. Although the angular velocity does not match a $\propto r^{-2}$ relation, which would correspond to conservation of the specific angular momentum within the computational domain, it is clearly a decreasing function of the radius, contrary to what is commonly assumed in 1D stellar evolution models. The profile we obtain is not described by any simple function, although a $10 \%$ quality fit can be obtained with a function of the form

$$
f(r)=a+b r^{n}
$$

with $\mathrm{a}=-141, \mathrm{~b}=122$ and $\mathrm{n}=-0.45$. On the plot in Fig. 3.b, we have also used a ratio of two polynomial functions in order to achieve a better fit (squares over the solid line). The complexity of the function mirrors the intricate combination of meridional circulation, viscosity and Reynolds stresses in transporting the angular momentum, and the important role of Reynolds stresses, which are the main competitor of meridional circulation. The sensitivity of this rotation law to various parameters of the simulation is currently under investigation (Brun \& Palacios 2006).

\section{Back to 1D modelling}

In this paper, we have presented the first simulation of turbulent rotating convection in the inner part of the extended convective envelope of a RGB low-mass star. This first case is moderately turbulent. The convection is strong, and structured in large cells that cover the top of the computational domain and become smaller at the base of the domain, in part due to geometrical effects. The developed convective state realized in our simulation is associated with very large temperature fluctuations and radial velocities, orders of magnitude larger than the ones found in similar simulations of the solar convection zone 
(Brun \& Toomre 2002). The differential rotation, which is nearly cylindrical, presents large gradients in both the latitudinal and radial directions. An average over all longitudes and latitudes reveals a mean radial profile far from the uniformity that has been generally assumed in 1D stellar evolution codes. For the present simulation, we obtain an acceptable fit using a much more intricate function than we would have expected in the case of conservation of the specific angular momentum.

Although we only explored one point in a large parameter space, which invites us to be cautious when drawing conclusions from this first run, the rotation law that emerges from the simulation presented here is much closer to the case of specific angular momentum conservation than to that of uniform rotation. This is consistent with what was expected from both observations and 1D models (see $\S 1$ ).

This encourages us to push further our investigation in order to be able to use the rotation profile predicted by the 3D simulation in 1D stellar evolution models (Palacios et al., in prep.).

\section{Acknowledgements}

A.P. acknowledges post-doctoral fellowship from the Centre d'Études Atomiques. Computations have been performed at CEA-CCRT and CNRS-IDRIS supercomputer centers.

\section{References}

Brun, A.S. \& Toomre, J. 2002, ApJ 570, 865

Brun, A.S., Miesch, M.S. \& Toomre, J. 2004, ApJ 614, 1073

Brun, A.S. \& Palacios, A. 2006, $A \mathscr{E} A$, submitted

Charbonnel, C. 1995, ApJL 453, L41

Charbonnel, C. \& do Nascimento, J. D. 1998, A\&A 336, 915

Gratton, R.G., Sneden, C., Carretta, E. \& Bragaglia, A. 2000, A\&A 354, 169

Palacios, A., Charbonnel, C., Talon, S. \& Siess, L. 2006, A\&A 453, 261

Sills, A. \& Pinsonneault, M. 2000, ApJ 540, 489

Sweigart A.V. \& Mengel J.G. 1979, ApJ 229, 624 (SM79)

\section{Discussion}

HANS-G. LuDWIG: In view of the stochastic excitation of pulsations: are pulsations present in your model?

A. Palacios: No. ASH is an Anelastic code, and acoustic modes are filtered.

F. KUPKA: Wouldn't it be necessary to run many more different simulations probing a much larger range of parameters before you can extract scaling laws that yo use in your $1 \mathrm{D}$ stellar evolution models?

A. Palacios: Yes, we're in the process of running further simulations, in particular a more turbulent simulation, but these are time consuming computations. The important point from these first results is that the rotation appears not to be uniform in the inner convective envelope of the red giant. This is different from the assumption that people normally do in stellar evolution. It is however encouraging because it seems to confirm the feeling that we had from observations, in particular from observations of the surface rotation velocities of horizontal branch stars.

Comment By F. Kupka: O.K, because from your conclusions I had the impression you're already in the process of putting you results back into the evolution code. 